Aug.12, 1998

\title{
The Eikonal Equation in Flat Space: Null Surfaces and Their Singularities. I
}

\author{
Simonetta Frittelli ${ }^{\mathrm{a}, \mathrm{b}}$, Ezra T. Newman ${ }^{\mathrm{b}}$, \\ Gilberto Silva-Ortigoza ${ }^{b, c}$ \\ ${ }^{a}$ Physics Department, Duquesne University, Pittsburgh, PA 15282, \\ ${ }^{\mathrm{b}}$ Department of Physics and Astronomy, \\ University of Pittsburgh, \\ Pittsburgh, PA 15260. \\ ${ }^{\mathrm{c}}$ Facultad de Ciencias Físico Matemáticas \\ de la Universidad Autónoma de Puebla, \\ Apartado Postal 1152, \\ 72001 Puebla, Pue., México.
}

\begin{abstract}
The level surfaces of solutions to the eikonal equation define null or characteristic surfaces. In this note we study, in Minkowski space, properties of these surfaces. In particular we are interested both in the singularities of these "surfaces" (which can in general self-intersect and be only piece-wise smooth) and in the decomposition of the null surfaces into a one parameter family of two-dimensional wavefronts which can also have self-intersections and singularities.

We first review a beautiful method for constructing the general solution to the flat-space eikonal equation; it allows for solutions either from arbitrary Cauchy data or for time independent (stationary) solutions of the form $S=t-S_{0}(x, y, z)$. We then apply this method to obtain global, asymptotically spherical, null surfaces that are associated with shearing ("bad") two-dimensional cuts of null infinity; the
\end{abstract}


surfaces are defined from the normal rays to the cut. This is followed by a study of the caustics and singularities of these surfaces and those of their associated wavefronts. We then treat the same set of issues from an alternative point of view, namely from Arnold's theory of generating families. This treatment allows one to deal (parametrically) with the regions of self-intersection and non-smoothness of the null surfaces, regions which are difficult to treat otherwise.

Finally, we generalize the analysis of the singularities to the case of families of characterisitic surfaces. 


\section{Introduction}

The study of the propagation of electromagnetic wavefronts, in the highfrequency or geometric optics limit, is almost ubiquitous in physics; it is a basic staple of elementary physics courses, it arises in the practical area of optical equipment, in applied subjects, too numerable to mention in detail, involving materials with variable refractive index , in atmospheric and astrophysical studies. They have been a prime illustration of V.I. Arnold's theory of Lagrangian and Legendre maps. 饮阳 In a different guise, similar problems arise in catastrophe theory. In addition to the various applications to more standard physics problems, they also play a most fundamental role in General Relativity, e.g., the continuous propagation of the two-dimensional wavefronts, i.e., the one parameter family of evolving wave fronts, form null (or characteristic) three-surfaces that are determined by the dynamics of the curved space-time in which the wavefronts propagate. 5 In this context it forms the basis for the theory of gravitational lensing. The converse statement is also true, namely that sets of null surfaces define, up to a conformal factor, the space-time geometry itself. 9 In arbitrary space-times, the high frequency limit is completely governed by the eikonal equation,

$$
g^{a b} \partial_{a} S_{b} S=0
$$

where $x^{a}=\left(x^{i}, t\right)$ are local space-time coordinates, $g^{a b}\left(x^{c}\right)$ is a given spacetime metric and $S=S\left(x^{a}\right)$. The level surfaces of $S$, i.e., $S\left(x^{a}\right)=$ constant, define the characteristic or null three-surfaces (or what Arnold calls the "big wave fronts" $\mathrm{B}$ ) and the $S\left(x^{i}, t\right)=$ constant for constant $t$ define the two dimensional "small" wavefront in the three dimensional space of $t=$ constant. The vector $p^{a}=g^{a b} \partial_{b} S$ is tangent to the null geodesics that rule the characteristic surface. Though we are basically interested in Eq.(1) for arbitrary space-times, here we will confine ourselves to a study of its solutions only in flat (Minkowski) space. (A future paper, in preparation, will generalize the present material to curved space-times.) Eq.(1) then becomes

$$
\eta^{a b} \partial_{a} S \partial_{b} S=\left(\partial_{t} S\right)^{2}-\left(\partial_{x} S\right)^{2}-\left(\partial_{y} S\right)^{2}-\left(\partial_{z} S\right)^{2}=0
$$

The level surfaces of the solutions to Eqs.(11) or (2), the null surfaces, can be viewed as being generated by the evolution of 2-dimensional wave fronts. Specifically, a wavefront evolves by following light-rays that are normal to it, generating the null three-surface. A smooth wavefront in three-space, in 
general progresses, into a singular one, either to the past or the future, i.e., a generic null surface in space-time has singularities. The singular wavefronts are 2-surfaces that are continuous with existing first derivative, but where (piece-wise) the second derivative are singular, being either undefined or infinite. The structure of the singularities are generically cusp ridges and swallowtails. There are unstable exceptions.

A textbook example胭 of flat space singular wavefronts (and associated big wavefront) are from imploding triaxial ellipsoids, where an initially ellipsoidal wavefront is evolved inwardly, it self-intersects for some finite period of time and eventually expands out to infinity, becoming spherical in the limit.

The singularities of wavefronts are also interpreted as the location of focusing regions, where the intensity of light becomes very high. At the focusing regions, neighboring null geodesics meet, and the cross sectional area of the bundle of light-rays collapses to zero, which leads to the increase in intensity. Spherical wavefronts focus at a single point, (which are unstable under small perturbations of the front) whereas generic wavefronts trace spatial curves of focusing points (cusp ridges and swallowtails).

In Section II we will review a beautiful method for giving the general solution to the flat-space eikonal; it allows for solutions either from arbitrary Cauchy data or for stationary solutions that arise from the ansatz, $S=$ $t-S_{0}(x, y, z)$.

In Sec. III, we will apply the method of Sec. II to obtain global asymptotically spherical null surfaces that are associated with shearing ("bad") cuts of null infinity, 10.11 They will be defined from the normal rays to a "bad" cut. This construction can be thought of as beginning with a deformed, initial, two-sphere in a finite region of space-time, then construct the future outward directed null normals to the two surface which generates a null surface and finally "slide" the initial two-surface along the null geodesics that generate the null surface, to future null infinity. This limit is the "bad" cut of null infinity.

In Sec. IV, we will study the caustics and singularities of these characteristic surfaces and their associated wavefronts.

In Sec. V, we treat the same problems of the singularities of these surfaces but now from an alternate point of view, namely from Arnold's theory of generating families. This treatment allows one to handle (parametrically) the regions of self-intersection and non-smoothness of the null surfaces.

In Sec. VI, we discuss a generalization of the ideas presented to this 
point. Though this generalization is primarily intended for use in non-flat Lorentzian space-times, nevertheless we believe that it is quite useful to see it in the simpler case of flat space-time; it allows for the clarification of certain points that would be difficult in more general situations. Specifically we will consider solutions of the eikonal equation that depend on two parameters that are different from the two parameter family of plane waves. We will see, the slightly surprising result, that the singularity structure of the individual characteristic surfaces can be studied via the parameter behavior of nearby solutions. More precisely, if the two parameter set of solutions is given by $Z\left(x^{a}, \mu, \bar{\mu}\right)$, the singularities of the level surfaces of $Z$ for fixed values of $(\mu, \bar{\mu})$ can be studied and expressed in terms of $(\mu, \bar{\mu})$ derivatives of $Z$. These results become important in asymptotically flat space-times where the $Z\left(x^{a}, \mu, \bar{\mu}\right)$ can be chosen to represent the family of past null cones from all the points of future null infinity.

\section{Solutions of the Eikonal Equation}

We review a powerful method for solving the flat-space eikonal equation with arbitrary given Cauchy data. We begin with a solution $S^{*}$ of the eikonal equation that depends on three arbitrary parameters, i.e.,

$$
S^{*}=S^{*}\left(x^{i}, t, \alpha_{i}\right)=x^{i} \alpha_{i}-t \sqrt{\Sigma\left(\alpha_{i}\right)^{2}}
$$

called a complete integral. A "general integral" (which involves an arbitrary function) can be constructed from the complete integral in the following manner: we first add to it an arbitrary function of the three $\alpha_{i}$, i.e., we consider

$$
S^{* *}=S^{*}\left(x^{i}, t, \alpha_{i}\right)-F\left(\alpha_{i}\right),
$$

with the weak condition that (aside from lower dimensional regions)

$$
\left|\frac{\partial^{2} S^{* *}}{\partial \alpha_{i} \partial \alpha_{j}}\right| \neq 0 .
$$

We next demand that $\partial S^{* *} / \partial \alpha_{i}=\partial S^{*} / \partial \alpha_{i}-\partial F / \partial \alpha_{i}=0$, which implies that there are three functions of the form $\alpha_{i}=A_{i}\left(x^{i}, t\right)$. (In general these solutions are not unique and they must be expressed on different sheets. See 
Sec. IV for a complete discussion of this issue.) Finally, via $\alpha_{i}=A_{i}\left(x^{i}, t\right)$, the $\alpha_{i}$ are eliminated in the $S^{* *}$ yielding (perhaps multivalued)

$$
S\left(x^{i}, t\right)=S^{*}\left(x^{i}, t, A_{i}\left(x^{i}, t\right)\right)-F\left(A_{i}\left(x^{i}, t\right)\right) .
$$

The level surfaces of this $S$ might self-intersect and be only piece-wise differentiable.

It is not difficult to show that the $S$, so constructed satisfies the eikonal equation.12 This follows immediately from the fact that

$$
\partial_{a} S=\partial_{a} S^{*}+\left(\partial_{A_{i}} S^{*}-\partial_{A_{i}} F\right) \partial_{a} A_{i}=\partial_{a} S^{*} .
$$

This solution now depends on an arbitrary function of three variables, namely the $F$. The task is now to determine the $F\left(\alpha_{i}\right)$ in terms of (appropriate) Cauchy data, $S_{C}\left(x^{i}\right)$. This is accomplished as follows; define $\alpha_{i}=$ $\partial S_{C} / \partial x^{i}$ and algebraically invert it in the form of the three equations $x^{i}=$ $X^{i}\left(\alpha_{i}\right)$. At $t=t_{0}$ we have that

$$
S\left(x^{i}, t_{0}\right)=S^{*}\left(x^{i}, t_{0}, A_{i}\left(x^{i}, t_{0}\right)\right)-F\left(A_{i}\left(x^{i}, t_{0}\right)\right) .
$$

Replacing all the $A_{i}$ by $\alpha_{i}$ and all the $x^{i}$ by $X^{i}\left(\alpha_{i}\right)$, we have that

$$
F\left(\alpha_{i}\right)=S^{*}\left(X^{i}, t_{0}, \alpha_{i}\right)-S_{C}\left(X^{i}\right),
$$

i.e., the free $F\left(\alpha_{i}\right)$ is now expressed in terms of the free Cauchy data, $S_{C}\left(x^{i}\right)$.

This allows us to find (in principle - modulo algebraic inversions or parametrizations) solutions of the flat-space eikonal equation with arbitrary Cauchy data.

There exists a special class of solutions that are not studied or found easily via the Cauchy problem, namely the "stationary" solutions which have the form

$$
S=t-S_{0}\left(x^{i}\right)
$$

To generate solutions of this form we modify the complete integral, Eq.3, making it a function of only two free parameters by imposing the condition that $\Sigma\left(\alpha_{i}\right)^{2}=$ constant; (for convenience chosen as $\Sigma\left(\alpha_{i}\right)^{2}=1 / \sqrt{2}$ ).

We then write the modified complete integral as

$$
S^{*}=x^{a} \ell_{a}(\zeta, \bar{\zeta})
$$

where 


$$
\ell_{a}(\zeta, \bar{\zeta})=\frac{1}{\sqrt{2}(1+\zeta \bar{\zeta})}[(1+\zeta \bar{\zeta}),-(\zeta+\bar{\zeta}),-i(\bar{\zeta}-\zeta),(1-\zeta \bar{\zeta})]
$$

The complex number $\zeta$, which plays the role of two of the independent parameters among the three $\alpha_{i}$, can be thought of as the complex stereographic coordinate on the sphere; the $\ell_{a}(\zeta, \bar{\zeta})$ is a Minkowski null vector, $\eta^{a b} \ell_{a} \ell_{b}=0$, that spans the entire lightcone as $\zeta$ ranges over the sphere. Eq.9 represents a spheres worth of different families of plane waves parametrized by the direction $\zeta$.

If we now take

$$
S^{* *}=x^{a} \ell_{a}(\zeta, \bar{\zeta})+\alpha(\zeta, \bar{\zeta})
$$

and construct $ð S^{* *}=\bar{\partial} S^{* *}=0$, i.e.,

$$
\begin{aligned}
& \omega \equiv x^{a} m_{a}(\zeta, \bar{\zeta})+ð \alpha(\zeta, \bar{\zeta})=0 \\
& \bar{\omega} \equiv x^{a} \bar{m}_{a}(\zeta, \bar{\zeta})+\bar{\partial} \alpha(\zeta, \bar{\zeta})=0
\end{aligned}
$$

where

$$
\begin{aligned}
& m_{a}(\zeta, \bar{\zeta})=\partial \ell_{a}(\zeta, \bar{\zeta}) \equiv(1+\zeta \bar{\zeta}) \frac{\partial \ell_{a}(\zeta, \bar{\zeta})}{\partial \zeta} \\
& \bar{m}_{a}(\zeta, \bar{\zeta})=\bar{\partial} \ell_{a}(\zeta, \bar{\zeta}) \equiv(1+\zeta \bar{\zeta}) \frac{\partial \ell_{a}(\zeta, \bar{\zeta})}{\partial \bar{\zeta}}
\end{aligned}
$$

For generic $\alpha(\zeta, \bar{\zeta})$, Eq.(12) can be solved for

$$
\zeta=\Upsilon(x, y, z),
$$

where again these solutions need not be unique and must be expressed on different sheets. (See Sec. IV for a full treatment of this problem.) Note that Eqs. (12) do not depend on the time $t$ and hence $\Upsilon$ is a function only of the spatial coordinates. When the $\Upsilon(x, y, z)$ is substituted into Eq.(11), i.e.,

$$
S(t, x, y, z)=\frac{t}{\sqrt{2}}-S_{0}(x, y, z)=\frac{t}{\sqrt{2}}+x^{i} \ell_{i}(\Upsilon, \bar{\Upsilon})+\alpha(\Upsilon, \bar{\Upsilon})
$$


we have a solution of the eikonal equation depending on an arbitrary function of two variables, $\alpha(\Upsilon, \bar{\Upsilon})$. The level surfaces of $S$ could in general self-intersect and be only piecewise differentiable.

The procedure of beginning with a complete solution and obtaining the general solution via the two (or three) variable arbitrary function $i s$ geometrically equivalent to the construction of an envelope from the family of plane waves as the two (or three) constants in the complete solutions are varied.13

Since in this work, we will only be interested in individual null surfaces and their properties, we can and will confine ourselves to the level surfaces of the solutions of the form given in Eq.(17).

\section{Null Surfaces Generated by Normals to Two-Surfaces}

We want to give a slightly different geometric interpretation to the method of the previous section for generating the stationary solutions of the eikonal equation. Given any (spatial) two-surface (for example consider any two dimensional slice of the past light cone of an arbitrary space-time point), the normal rays to the surface (either the outgoing or incoming ones) generate a null surface. In this section we will consider a particular case of this construction where this past lightcone is taken to be the future null infinity, $\mathfrak{I}^{+}$, of Penrose. 10

The future null boundary, $\mathfrak{I}^{+}$, (the endpoints of future directed null geodesics) of any asymptotically simple space-time is a null surface with topology $R \times S^{2}$. A choice of Bondi coordinates $(u, \zeta, \bar{\zeta})$ can be assigned to $\mathfrak{I}^{+}$, where $\zeta=\cot (\theta / 2) e^{i \phi}$ for the $S^{2}$ sector. The intersection of the future lightcone $\mathfrak{C}_{x}$ of a point $x^{a}$ with $\mathfrak{I}^{+}$is a two-surface, locally imbedded in $R \times S^{2}$; it can generically be described locally by $u=Z\left(x^{a}, \zeta, \bar{\zeta}\right)$. The twosurface is referred to as a lightcone cut, 14 whereas the function $Z\left(x^{a}, \zeta, \bar{\zeta}\right)$ is referred to as a lightcone cut function, and is a two-point real function on the space-time and the boundary, $\mathfrak{I}^{+}$.

Remark 1 Though for this work it is irrelevant, we mention that the lightcone cut function $Z\left(x^{a}, \zeta, \bar{\zeta}\right)$ is one of two fundamental variables in a reformulation of general relativity via null surfaces. information of the space-time.. 
In the remainder of this work we will confine ourselves to flat space-time where (modulo Poincare transformations) a natural choice of Bondi coordinates $\left(u_{n}, \zeta, \bar{\zeta}\right)$ exists; the $u_{n}=$ constant is constructed from the intersection of the future lightcone, $\mathfrak{C}_{(t, o, o, o)}$, of the spatial origin, at time $t=u_{n}$, with $\mathfrak{I}^{+}$; the $(\zeta, \bar{\zeta})$ are just the null directions, at the origin, carried along by the null generators of the lightcone. Using Cartesian coordinates $x^{a}$ for the space-time and these natural Bondi coordinates, 15 the lightcone cuts can be described as

$$
u_{n}=x^{a} \ell_{a}(\zeta, \bar{\zeta})
$$

where $\ell_{a}$ represents the covariant version of a null vector $\ell^{a}$ with Cartesian components given as

$$
\begin{aligned}
\ell^{a}(\zeta, \bar{\zeta}) & =\frac{1}{\sqrt{2}(1+\zeta \bar{\zeta})}((1+\zeta \bar{\zeta}),(\zeta+\bar{\zeta}), i(\bar{\zeta}-\zeta),(\zeta \bar{\zeta}-1)) \\
& =\frac{1}{\sqrt{2}}(1, \sin \theta \cos \phi, \sin \theta \sin \phi, \cos \theta)
\end{aligned}
$$

Adding the radial coordinate $r$, this natural choice of Bondi coordinates is identical to the standard null polar coordinates $\left(u_{n}, r, \zeta, \bar{\zeta}\right)$ given by

$$
x^{a}=u t^{a}+r \ell^{a}(\zeta, \bar{\zeta}), t^{a}=\sqrt{2}(1,0,0,0)
$$

Note that Eqs.(18) and (19) are identical with Eqs.(9) and (10) though their meanings are different; Eq.(18) has the dual meaning of being, for fixed value of the $x^{a}$, the lightcone cut of $\mathfrak{I}^{+}$and also, for fixed values of $\left(u_{n}, \zeta, \bar{\zeta}\right)$, it describes the plane wave (null surface) intersecting the time axis at $t=u_{n}$ in the direction of $(\zeta, \bar{\zeta})$.

By Eq.(18), the lightcone cuts of any points $x^{a}=(t, 0,0,0)$ along the time axis take, as we mentioned earlier, a constant value on $\mathfrak{I}^{+}$, namely, they are the constant- $u_{n}$ slices. The natural Bondi cuts are lightcone cuts as well. By following inwardly the null geodesics that leave the natural Bondi cuts orthogonally, we find no focusing other than at the apex (on the time axis) of the lightcone.

By a slight modification of the above we can find other null surfaces leaving $\mathfrak{I}^{+}$that have much more complicated focusing properties than that of a lightcone. If we consider the one parameter family of cuts of $\mathfrak{I}^{+}$given, say by,

$$
u_{n}=-\alpha(\zeta, \bar{\zeta})+u
$$


where $\alpha(\zeta, \bar{\zeta})$ is a given but arbitrary regular function on $S^{2}$, and $u$ is a parameter on $R$, we can ask for the null surfaces generated by the null normals to the family of cuts.

- Note that Eq.(21) can be rewritten as $u=u_{n}+\alpha(\zeta, \bar{\zeta})$ and reinterpreted as a (Bondi-Metzner-Sachs) supertranslation 11 between the coordinates $u_{n}$ and $u$ on $\mathfrak{I}^{+}$.

We now construct the null surface formed by the normal rays to the cuts, Eq.(21), determined by $u=$ const.; replacing the $u_{n}$ in Eq.(21) by the null planes Eq.(18), $\left[u_{n}=x^{a} \ell_{a}(\zeta, \bar{\zeta})\right]$, we have

$$
u=x^{a} \ell_{a}(\zeta, \bar{\zeta})+\alpha(\zeta, \bar{\zeta})
$$

which is identical to Eq.(11). The envelope formed from all the null planes that are normal to the family of cuts are found by setting to zero the $\zeta$ and $\bar{\zeta}$ derivatives of Eq. (22) and eliminating the $(\zeta, \bar{\zeta})$ from (22), a procedure identical to that followed in the previous section to obtain Eq.(17), i.e., we now have the one parameter family of null surfaces

$$
S^{* *} \equiv u=\frac{t}{\sqrt{2}}-x^{i} \ell_{i}(\Upsilon, \bar{\Upsilon})+\alpha(\Upsilon, \bar{\Upsilon})
$$

with $\zeta=\Upsilon(x, y, z)$, a solution of Eqs.(12),(13). The procedure of setting to zero the $\omega$ and $\bar{\omega}$ in

$$
\begin{aligned}
& \omega \equiv x^{a} m_{a}(\zeta, \bar{\zeta})+ð \alpha(\zeta, \bar{\zeta})=0 \\
& \bar{\omega} \equiv x^{a} \bar{m}_{a}(\zeta, \bar{\zeta})+\bar{\partial} \alpha(\zeta, \bar{\zeta})=0
\end{aligned}
$$

selects the null ray at each point of $\mathfrak{I}^{+}$that is orthogonal to the cut given by Eq.(21).

- Note that $\alpha$ can be chosen to contain only spherical harmonics of order $l>2$ since any $l=0,1$ components of $\alpha$ could be absorbed by $x^{a} \ell_{a}$ with no modification other than displacing the origin of the coordinates $x^{a}$, since $\ell_{a}$ is precisely the collection of spherical harmonics of order 0 and 1. 
We can give a parametric description of the family of null surfaces, Eq.(23), by the following procedure: we consider the four functions

$$
\begin{gathered}
\zeta=\Upsilon(x, y, z) \Leftrightarrow x^{a} m_{a}(\zeta, \bar{\zeta})+\partial \alpha(\zeta, \bar{\zeta})=0 \\
\bar{\zeta}=\bar{\Upsilon}(x, y, z) \Leftrightarrow x^{a} \bar{m}_{a}(\zeta, \bar{\zeta})+\bar{\partial} \alpha(\zeta, \bar{\zeta})=0 \\
u=u(t, x, y, z)=\left.\left\{x^{a} \ell_{a}(\zeta, \bar{\zeta})+\alpha(\zeta, \bar{\zeta})\right\}\right|_{\Upsilon, \bar{\Upsilon}} \\
r=r(x, y, z)=\left.\left\{x^{a}\left(n_{a}-\ell_{a}\right)(\zeta, \bar{\zeta})+\partial \bar{\partial} \alpha(\zeta, \bar{\zeta})\right\}\right|_{\Upsilon, \Upsilon}
\end{gathered}
$$

and consider them as a coordinate transformation between the $\{u, \zeta, \bar{\zeta}, r\}$ and the $\left\{x^{a}\right\}$. We have used $m^{a} \equiv \partial \ell^{a}, \bar{m}^{a} \equiv \bar{\partial} \ell^{a}$, and $n^{a} \equiv \partial \bar{\partial} \ell^{a}+\ell^{a}$. From the fact that $\left(\ell^{a}, m^{a}, \bar{m}^{a}, n^{a}\right)$ form a null tetrad for every fixed value of $(\zeta, \bar{\zeta})$, this coordinate transformation can be readily inverted into the form

$$
x^{a}=(u-\alpha)\left(n^{a}+\ell^{a}\right)+(r-\partial \bar{\partial} \alpha) \ell^{a}+(ð \alpha) \bar{m}^{a}+(\bar{\partial} \alpha) m^{a}
$$

This relationship can alternatively be looked on as the parametric version of the one-parameter family of null surfaces, Eq.(23), (where, for fixed $u$, the $(r, \zeta, \bar{\zeta})$ parametrize the surface) or as the coordinate transformation between the $\left\{x^{a}\right\}$ and the null-geodesic coordinates, $(u, \zeta, \bar{\zeta}, r)$; $u$ labels the null surfaces, the pair $(\zeta, \bar{\zeta})$ labels null geodesics (via their intersection with $\mathfrak{I}^{+}$) and $r$ is an affine parameter along the null geodesics. That this is true can be easily seen from the parametric form, Eq. (30), by simply constructing

$$
\frac{d x^{a}}{d r}=\ell^{a}(\zeta, \bar{\zeta})
$$

and observing that $\ell^{a}$ is a null tangent vector with affine normalization.

The transformation between the $x^{a}$ and the $(u, r, \zeta, \bar{\zeta})$ breaks down when the Jacobian of the transformation, Eq.(30), vanishes, i.e., when

$$
D \equiv \frac{\partial(t, x, y, z)}{\partial(u, r, \zeta, \bar{\zeta})}=r^{2}-\partial^{2} \alpha \bar{\partial}^{2} \alpha=0 .
$$

Geometrically, this is where the null surface develops singularities. In the projection to the three-space $(x, y, z)$ it is a two surface; the "caustic surface". 
To see this explicitly, we return to Eq.(30) where we have (for fixed $u$ ) that $(x, y, z)=x^{i}=X^{i}(r, \zeta, \bar{\zeta})$, i.e., are known functions of $(r, \zeta, \bar{\zeta})$. If the $r$ in $X^{i}$ is replaced by the $r$ from Eq. (32) we have the parametric form of the caustic,

$$
x^{i}=X^{i}(r(\zeta, \bar{\zeta}), \zeta, \bar{\zeta})=\widehat{X}^{i}(\zeta, \bar{\zeta}) .
$$

We will return to this in the next section.

It is interesting to note that the coordinates $(u, \zeta, \bar{\zeta}, r)$ represent a type of null coordinate system that we could call asymptotic null-polar coordinates which are the flat space case of an interior Bondi coordinate system, 16,11 i.e., the extension into the interior of the space-time of the Bondi coordinates $(u, \zeta, \bar{\zeta})$ on $\mathfrak{I}^{+}$. They differ from the standard null polar coordinates by the fact that the null geodesics that rule these surfaces possess non-vanishing shear while for the standard ones the shear vanishes.

The complex shear is defined as $\sigma=M^{a} M^{b} \nabla_{a} L_{b}$, where $L_{b}$ is tangent to the null geodesics and $M^{a}$ is complex null, orthogonal to $L_{b}$ and such that $M^{a} \bar{M}_{a}=-1$. In our case, because of Eqs.(30) and (31) and the fact that $\left(\ell^{a}, m^{a}, \bar{m}^{a}, n^{a}\right)$ forms a null tetrad, we have that $L_{b}=\ell_{b}$ and $M^{a}=m^{a}$. Furthermore, the gradient of $\ell_{b}$ is $\nabla_{a} \ell_{b}=m_{a} \zeta_{, b}+\bar{m}_{a} \bar{\zeta}_{, b}$ and thus $\sigma=-m^{b} \bar{\zeta}_{, b}$. To obtain the derivative of $\bar{\zeta}$ along $m^{b}$ we take the gradients of Eqs.(24) and (25) which yields

$$
\begin{aligned}
& m_{b}(\zeta, \bar{\zeta})+\left\{x^{a}\left(n_{a}-l_{a}\right)+\partial \bar{\partial} \alpha\right\} \bar{\zeta}_{, b}+\chi^{2} \alpha \zeta_{, b}=0 \\
& \bar{m}_{b}(\zeta, \bar{\zeta})+\left\{x^{a}\left(n_{a}-l_{a}\right)+ð \bar{\partial} \alpha\right\} \zeta_{, b}+\bar{\chi}^{2} \alpha \bar{\zeta}_{, b}=0 .
\end{aligned}
$$

Using Eq.(29) and contracting Eqs. (34) and (35) by $m^{b}$, we obtain

$$
\begin{aligned}
r m^{b} \bar{\zeta}_{, b}+\chi^{2} \alpha m^{b} \zeta_{, b} & =0 \\
-1+r m^{b} \zeta_{, b}+\bar{\partial}^{2} \alpha m^{b} \bar{\zeta}_{, b} & =0 .
\end{aligned}
$$

By eliminating the $m^{b} \zeta_{, b}$ from these equations, we find

$$
\left(r^{2}-ð^{2} \alpha \bar{\partial}^{2} \alpha\right) m^{b} \bar{\zeta}_{, b}+\gamma^{2} \alpha=0
$$

or

$$
\sigma=\frac{\sigma^{0}}{r^{2}-\sigma^{0} \bar{\sigma}^{0}}
$$


where $\sigma^{0}=\gamma^{2} \alpha$. This is also a confirmation of the Sachs theorem on the transformation of the asymptotic shear, $\sigma^{0}$, under a BMS transformation. Eq.(39) represents a special (non-twisting) case of a more general result valid for generic null congruences in flat space 17

We point out that the flat-space line element, using Eq.(30), can easily be expressed in terms of these shearing Bondi coordinates as 18

$$
\begin{aligned}
d s^{2}= & \eta_{a b} d x^{a} d x^{b} \\
= & 2 d u\left(d u+d r-\bar{\partial} \partial^{2} \alpha \frac{d \zeta}{P}-\partial \bar{\partial}^{2} \alpha \frac{d \bar{\zeta}}{P}\right) \\
& -\frac{2 r}{P^{2}}\left(\partial^{2} \alpha d \zeta^{2}+\bar{\partial}^{2} \alpha d \bar{\zeta}^{2}\right)-2\left(r^{2}+\jmath^{2} \alpha \bar{\partial}^{2} \alpha\right) \frac{d \zeta d \bar{\zeta}}{P^{2}}
\end{aligned}
$$

where $P=1+\zeta \bar{\zeta}$. The determinant of $g_{a b}$ is given by $|g|=\frac{1}{P^{4}}\left\{r^{2}-\right.$ $\left.\partial^{2} \alpha \bar{\partial}^{2} \alpha\right\}^{2}=\frac{D^{2}}{P^{4}}$ whose vanishing agrees with the vanishing of the Jacobian, Eq. (32).

Note that the asymptotic $r=$ const. $\Rightarrow \infty$ sections, at $u=$ const., become metric spheres.

- It is perhaps interesting to speculate on the use of Eq. (40) as the Minkowski space lowest order term, in perturbation calculations, for solutions of the Einstein equations.

We complete this section by showing how a null surface can be constructed explicitly from the normals to an arbitrary space-like two surface, $\mathfrak{S}$, in a manner virtually identical to those constructed from a cut or slice of null infinity.

We begin from Eq. (22)

$$
u=0=x^{a} \ell_{a}(\zeta, \bar{\zeta})+\alpha(\zeta, \bar{\zeta})
$$

with Eqs. 24 and 25). The issue is, given the surface $\mathfrak{S}$, how is one to choose $\alpha(\zeta, \bar{\zeta})$ ?

First $\mathfrak{S}$ is defined parametrically, $x^{a}=x_{0}^{a}(\zeta, \bar{\zeta})$, where the parameters are chosen as follows: consider a time-like world line at the spacial origin and the family of light-cones centered on the line. The null geodesics ruling these cones are labeled by their directions $(\zeta, \bar{\zeta})$ on the sphere and coincide with labeling of the generators of null infinity. The points on $\mathfrak{S}$ are now (locally) 
parametrized by the labels of the null geodesics passing thru those points. With this parametrization the function $\alpha(\zeta, \bar{\zeta})$ is defined by

$$
\alpha(\zeta, \bar{\zeta})=-x_{0}^{a}(\zeta, \bar{\zeta}) \ell_{a}(\zeta, \bar{\zeta})
$$

so that Eq. (23) becomes

$$
u=0=\left(x^{a}-x_{0}^{a}(\zeta, \bar{\zeta})\right) \ell_{a}(\zeta, \bar{\zeta})
$$

and Eqs.(24 and 25) become

$$
\left(x^{a}-x_{0}^{a}(\zeta, \bar{\zeta})\right) m_{a}(\zeta, \bar{\zeta})-\ell_{a}(\zeta, \bar{\zeta}) ð x_{0}^{a}=0 \text { and c.c. }
$$

We see that the null surface so defined goes thru $\mathfrak{S}$, i.e., thru $x^{a}=x_{0}^{a}(\zeta, \bar{\zeta})$. To see that it is also normal to $\mathfrak{S}$, we notice that at $\mathfrak{S}$ the first two terms of Eq.(41) cancel out and we are left at $\mathfrak{S}$ with

$$
\ell_{a}(\zeta, \bar{\zeta}) ð x_{0}^{a}=0
$$

Thus as was claimed, the tangent vectors to $\mathfrak{S}$, namely $\partial x_{0}^{a}(\zeta, \bar{\zeta})$, are normal to the null tangent vectors to the null surface, $\ell_{a}$.

We see that the earlier construction of null surfaces from cuts of null infinity actually includes those constructed from finite surfaces.

\section{Wavefront evolution and singularities}

In the previous section, we mentioned that the null coordinate system broke down and the associated shearing null surfaces developed caustics at the points where $r^{2}=\partial^{2} \alpha \bar{\partial}^{2} \alpha$.

Here we focus our attention on the two dimensional wavefronts associated with these null surfaces. We show that the wavefronts develop singularities, and we locate the singularities via the standard method of singularity theory, and via our lightcone cut approach. The evolution of these singularities as the wave fronts evolve become the caustics.

A wavefront is, by definition, the intersection of our null surface, $u=u_{0}$, with a constant-time $t_{0}$ surface. This represents an instant in the progression of a wave. In our case, this requires fixing the time coordinate $x^{0}=t_{0}$ in Eq.(30), and solving for

$$
r=\sqrt{2} t_{0}-2 u_{0}+2 \alpha+\gamma \bar{\delta} \alpha
$$


The remaining coordinates $(x, y, z)$, using Eq. (42) to eliminate $r$, trace a twosurface (a "small" wave front) in the Euclidean three-space, parametrized by $(\zeta, \bar{\zeta})$ [or $(\theta, \phi)$ under the transformation $\left.\zeta=e^{i \phi} \cot \theta / 2\right]$ :

$$
\begin{aligned}
& x=\frac{1}{\sqrt{2}}\left[\left(\sqrt{2} t_{0}-2 u_{0}+2 \alpha\right) \frac{(\zeta+\bar{\zeta})}{(1+\zeta \bar{\zeta})}+\bar{\partial} \alpha \frac{\left(1-\bar{\zeta}^{2}\right)}{(1+\zeta \bar{\zeta})}+ð \alpha \frac{\left(1-\zeta^{2}\right)}{(1+\zeta \bar{\zeta})}\right] \\
& y=\frac{i}{\sqrt{2}}\left[\left(\sqrt{2} t_{0}-2 u_{0}+2 \alpha\right) \frac{(\bar{\zeta}-\zeta)}{(1+\zeta \bar{\zeta})}-\bar{\partial} \alpha \frac{\left(1+\bar{\zeta}^{2}\right)}{(1+\zeta \bar{\zeta})}+ð \alpha \frac{\left(1+\zeta^{2}\right)}{(1+\zeta \bar{\zeta})}\right] \\
& z=\frac{1}{\sqrt{2}}\left[\left(\sqrt{2} t_{0}-2 u_{0}+2 \alpha\right) \frac{(\zeta \bar{\zeta}-1)}{(1+\zeta \bar{\zeta})}+\bar{\partial} \alpha \frac{2 \bar{\zeta}}{(1+\zeta \bar{\zeta})}+ð \alpha \frac{2 \zeta}{(1+\zeta \bar{\zeta})}\right]
\end{aligned}
$$

The map $(\zeta, \bar{\zeta}) \rightarrow(x, y, z)$ is singular at points where the Jacobian matrix

$$
\left(\begin{array}{ll}
\partial x & \bar{\partial} x \\
\partial y & \bar{\partial} y \\
\partial z & \bar{\partial} z
\end{array}\right)
$$

drops rank, from 2 to 1 or 0 . The drop in rank takes place if the three 2-determinants vanish simultaneously:

$$
\begin{aligned}
& ð x \bar{\partial} y-\partial y \bar{\partial} x=0, \\
& \partial y \bar{\partial} z-ð z \bar{\partial} y=0, \\
& \partial z \bar{\partial} x-\partial x \bar{\partial} z=0 .
\end{aligned}
$$

Since

$$
\begin{aligned}
& \left.\partial^{a}\right|_{u, t}=\partial^{2} \alpha \bar{m}^{a}+r m^{a}, \\
& \left.\bar{\partial} x^{a}\right|_{u, t}=\bar{\partial}^{2} \alpha m^{a}+r \bar{m}^{a},
\end{aligned}
$$

the explicit expressions of the 2-determinants are as follows

$$
\begin{aligned}
& ð x \bar{\partial} y-\partial y \bar{\partial} x=\left(r^{2}-\jmath^{2} \alpha \bar{\partial}^{2} \alpha\right)\left(m^{x} \bar{m}^{y}-\bar{m}^{x} m^{y}\right), \\
& \text { ðy } \bar{\partial} z-ð z \bar{\partial} y=\left(r^{2}-\partial^{2} \alpha \bar{\partial}^{2} \alpha\right)\left(m^{y} \bar{m}^{z}-\bar{m}^{y} m^{z}\right) \text {, } \\
& \text { ð } z \bar{\partial} x-\partial x \bar{\partial} z=\left(r^{2}-\partial^{2} \alpha \bar{\partial}^{2} \alpha\right)\left(m^{z} \bar{m}^{x}-\bar{m}^{z} m^{x}\right) \text {. }
\end{aligned}
$$

Thus, all three determinants vanish at points where

$$
D \equiv r^{2}-\chi^{2} \alpha \bar{\partial}^{2} \alpha=0
$$


which, with $r$ from Eq.(42), determines a curve, the wavefront singularities. Note that the evolution of the wavefront singularities (obtained by varying $\left.t_{0}\right)$ yields the caustic surface.

Since $\alpha$ is a regular function on the sphere, so is $\gamma^{2} \alpha \bar{\delta}^{2} \alpha$; therefore, Eq.(55) (with $r$ given by Eq.(42)) admits solutions $\zeta$ only within a finite interval of time $t$. Thus the wavefronts are singular only during a closed interval of time. On the other hand, at very long times the wavefronts become spherical, which follows from the line element Eq. (40).

The wavefront singularities (curves) are places where neighboring null geodesics meet. We have a null surface $x^{a}(r, \zeta, \bar{\zeta})$ foliated by null geodesics. At every fixed value of $r$, there are two connecting vectors $\left.\partial x^{a}\right|_{u, r}$ and $\left.\bar{\partial} x^{a}\right|_{u, r}$. The null geodesics in this congruence meet wherever the area orthogonal to the congruence, spanned by the connecting vectors, vanishes. The connecting vectors are, explicitly,

$$
\begin{aligned}
& \left.\partial x^{a}\right|_{u, r}=-\bar{\partial} \partial^{2} \alpha \ell^{a}+\partial^{2} \alpha \bar{m}^{a}+r m^{a}, \\
& \left.\bar{\partial} x^{a}\right|_{u, r}=-\partial \bar{\partial}^{2} \alpha \ell^{a}+r \bar{m}^{a}+\bar{\partial}^{2} \alpha m^{a},
\end{aligned}
$$

The area spanned by the connecting vectors (calculated from their skew product) is simply $D \equiv r^{2}-\partial^{2} \alpha \bar{\partial}^{2} \alpha$. The vanishing of this area takes place at exactly the points given by Eq. (55).

We close this section with two examples.

Example 1: $\alpha=Y_{20}=3 \cos ^{2} \theta-1$. Due to axial symmetry, the wavefronts and their singularities for this choice of $\alpha$ can be completely worked out analytically, which gives insights into more general cases. The wavefronts at a given time $t$ are given by

$$
\begin{aligned}
& x=\frac{1}{\sqrt{2}} \sin \theta \cos \phi\left(\sqrt{2} t-2 u-2-6 \cos ^{2} \theta\right), \\
& y=\frac{1}{\sqrt{2}} \sin \theta \sin \phi\left(\sqrt{2} t-2 u-2-6 \cos ^{2} \theta\right), \\
& z=\frac{1}{\sqrt{2}} \cos \theta\left(\sqrt{2} t-2 u+10-6 \cos ^{2} \theta\right) .
\end{aligned}
$$

These are axially symmetric. For a closed interval of time, all the wavefronts are singular. For early and late times, however, the wavefronts are smooth. 
The singular points are located by eliminating $r$ from Eqs.(55) and (42), yielding in this case, the two solutions or "sheets"

$$
\left(\sqrt{2} t-2 u+10-18 \cos ^{2} \theta\right)\left(\sqrt{2} t-2 u-2-6 \cos ^{2} \theta\right)=0 .
$$

There is a solution $\theta$ only at times $\sqrt{2}(u-5) \leq t \leq \sqrt{2}(4+u)$. This is the interval where every wavefront is singular. A smooth wavefront and its corresponding profile at a late time are shown in Fig. 1. A wavefront at a time when both the cusp ridge singularities and the $z$-axis singularities are occurring, and its corresponding profile, are shown in Fig. 2. In Fig. 3, we have a later wavefront and profile with only the cusp ridge singularity. In Fig. 4, we display the evolution of the singularities forming the caustic 2-surface.

The high symmetry of this case is responsible for the lack of resemblance of the singular points on the $z$-axis with standard cusps. At these points, null geodesics labeled by different, but neighboring values of $\phi$, meet. This is clear from the fact that $\partial x / \partial \phi=\partial x / \partial \phi=\partial z / \partial \phi=0$ at these points, therefore the vector that connects geodesics with different values of $\phi$ vanishes.

In order to make a comparison, Fig. 5 shows a wavefront in the evolution of an imploding ellipsoid of revolution which is very similar to that of Example 1. In this case, an ellipsoid of revolution sends an incoming wavefront, which develops singularities during a certain interval of time. The standard cuspoidal ridges are clearly visible as rings at both ends of the figure. However, the crossover points in between are also singular, of the same type of singularity as that one developed in our example. Assuming a speed of light of 1 , the formulas for the imploding wavefront in this case are

$$
\begin{aligned}
& x=a \sin \theta \cos \phi\left(1-\frac{t}{\sqrt{a^{2} \sin ^{2} \theta+\left(a^{2} / c\right)^{2} \cos ^{2} \theta}}\right) \\
& y=a \sin \theta \sin \phi\left(1-\frac{t}{\sqrt{a^{2} \sin ^{2} \theta+\left(a^{2} / c\right)^{2} \cos ^{2} \theta}}\right) \\
& z=c \cos \theta\left(1-\frac{t}{\sqrt{\left(c^{2} / a\right)^{2} \sin ^{2} \theta+c^{2} \cos ^{2} \theta}}\right)
\end{aligned}
$$

Example 2: $\alpha=\operatorname{Real}\left(Y_{21}\right)=(\zeta+\bar{\zeta})(\zeta \bar{\zeta}-1) /(1+\zeta \bar{\zeta})^{2}$. In this case, there is no advantage in writing the wavefronts explicitly. 
However, they can be plotted with ease, displaying the typical singularities of three-dimensional wavefronts, namely swallowtails and cusp ridges. Cusp ridges are clearly visible in Fig. 6, which represents a wavefront at $u=0$ and $t=1.5$. Swallow tails are exemplified in Fig.7, which represents another wavefront in the same $u=0$ family, but at a later time of $t=2.35$. Locally all swallowtail have the form of Fig.8.

Both Figs. 6 and 7 compare remarkably well with a typical wavefront in the evolution of a triaxial ellipsoid, in which case an ellipsoid $(x / a)^{2}+(y / b)^{2}+(z / c)^{2}=1$ emits a wavefront of light inwardly, which develops singularities for a period of time. A typical singular imploding wavefront is shown in Fig. 9. The formulas for the imploding triaxial-ellipsoidal wavefront are the following:

$$
\begin{aligned}
& x=a \sin \theta \cos \phi\left(1-\frac{t}{a^{2} \sqrt{\left(\sin \theta \cos \phi / a^{2}\right)^{2}+\left(\sin \theta \sin \phi / b^{2}\right)^{2}+\left(\cos \theta / c^{2}\right)^{2}}}\right) \\
& y=b \sin \theta \sin \phi\left(1-\frac{t}{b^{2} \sqrt{\left(\sin \theta \cos \phi / a^{2}\right)^{2}+\left(\sin \theta \sin \phi / b^{2}\right)^{2}+\left(\cos \theta / c^{2}\right)^{2}}}\right) \\
& z=c \cos \theta\left(1-\frac{t}{c^{2} \sqrt{\left(\sin \theta \cos \phi / a^{2}\right)^{2}+\left(\sin \theta \sin \phi / b^{2}\right)^{2}+\left(\cos \theta / c^{2}\right)^{2}}}\right)
\end{aligned}
$$

\section{Generating Families}

In this section we will study the subject of the caustics of the null surfaces and the wavefront singularities via an alternative method, namely from the use of generating families for the construction of Lagrangianand Legendre submanifolds (developed by V. I. Arnold and his colleagues 2 2.6) associated with cotangent and contact bundles over space-time. The value of this treatment is that it allows one to deal (via a parametric representation) with the regions of self-intersection and non-differentiability of the null surfaces.

We first give a brief review of a special case of this theory that is adapted to the problem of null surfaces in four-dimensional space-time. Consider a four dimensional Lorentzian manifold, (with local coordinates, $\left\{t, x^{i}\right\}$ ) foliated by the constant $t$ surfaces. Now consider the $x^{i}$ as the coordinates of a configuration space $M$ and $p_{i}$ as the conjugate momentum so that we have the six dimensional cotangent bundle $T^{*} M$, with local coordinates $\left(x^{i}, p_{i}\right)$. 
We now describe the construction of a three dimensional submanifold of $T^{*} M$ (a Lagrangian submanifold - a maximal submanifold such that the symplectic form, restricted to it, vanishes) that plays a fundamental role in the discussion of the singularities of wavefronts and their associated caustics. We begin with a general description without any particular choice of dynamics, later restricting ourselves to null geodesic motion.

Choose a scalar function (determined later from the dynamics), referred to as a generating family, of the six variables

$$
f=F\left(t, x^{i}, \zeta, \bar{\zeta}\right)
$$

the $x^{i}$ are spatial points, the $(\zeta, \bar{\zeta})$ are parametric labels (for convenience we are using a complex representation) for points on a given spatial two-surface, $\mathfrak{s}$, i.e., we have a two-point function, while $t$ is the time for the (dynamic) particle to go from a point on $\mathfrak{s}$ to the point $x^{i}$. For a constant value of $f$, we consider Eq.(66) as defining $t$ implicitly as a function of $\left(x^{i}, \zeta, \bar{\zeta}\right)$, i.e.,

$$
t=T\left(f ; x^{i}, \zeta, \bar{\zeta}\right)
$$

or simply

$$
t=T\left(x^{i}, \zeta, \bar{\zeta}\right)
$$

Note that $T$ might be a multivalued function of its arguments, in which case it must be considered separately on the different sheets.

We now ask for the relationship between the $\left(x^{i}, \zeta, \bar{\zeta}\right)$ when $T$ is an extremal under variations of the $(\zeta, \bar{\zeta})$; i.e., we require that

$$
\partial T / \partial \zeta=\partial T / \partial \bar{\zeta}=0
$$

which in turn forces

$$
\partial F / \partial \zeta=\partial F / \partial \bar{\zeta}=0
$$

Finally a rank condition is imposed on the choice of $F$; the following $2 \times 5$ matrix must have rank two

$$
\left[\begin{array}{ccc}
\frac{\partial^{2} F}{\partial \zeta^{2}}, & \frac{\partial^{2} F}{\partial \zeta \partial \bar{\zeta}}, & \frac{\partial^{2} F}{\partial \zeta \partial x^{i}} \\
\frac{\partial^{2} F}{\partial \zeta \partial \bar{\zeta}}, & \frac{\partial^{2} F}{\partial \bar{\zeta}^{2}}, & \frac{\partial^{2} F}{\partial \bar{\zeta} \partial x^{i}}
\end{array}\right] .
$$

The meaning of this condition is that the two equations (69 or 68) can be solved (locally) in at least one of a variety of possible ways for two of the five 
variables $\left(x^{i}, \zeta, \bar{\zeta}\right)$; often it is necessary to solve them in the different ways in different regions. We then have three different possible cases:

1. $\zeta=\Upsilon\left(x^{i}\right), \bar{\zeta}=\bar{\Upsilon}\left(x^{i}\right)$; the simplest of the three cases. It allows $F$ to be treated as a function of just $x^{i}$. In the other cases $F$ must be treated parametrically.

2. $\zeta=\Psi\left(x^{A}, \bar{\zeta}\right), x^{J}=X^{J}\left(x^{A}, \bar{\zeta}\right)$, where $x^{A}$ are any two of the three $x^{i}$ and $x^{J}$ is the third one - or the conjugate version, $\bar{\zeta}=\bar{\Psi}\left(x^{A}, \zeta\right), x^{J}=$ $X^{J}\left(x^{A}, \zeta\right)$.

3. $x^{A}=X^{A}\left(x^{J}, \zeta, \bar{\zeta}\right)$, where again $x^{A}$ are any two of the three $x^{i}$ and $x^{J}$ is the third one.

Case 1 can occur when the determinant

$$
\hat{D}=\left|\begin{array}{ll}
\frac{\partial^{2} F}{\partial \zeta^{2}} & \frac{\partial^{2} F}{\partial \zeta \partial \bar{\zeta}} \\
\frac{\partial^{2} F}{\partial \zeta \partial \bar{\zeta}} & \frac{\partial^{2} F}{\partial \bar{\zeta}^{2}}
\end{array}\right| \neq 0 ;
$$

$\hat{D}=0$ when there is a critical point of the Lagrangian map defined shortly.

The Lagrangian submanifold obtained from $F$ is defined in the following way:

First we have $p_{i}=\frac{\partial F}{\partial x^{i}}$. (Note that this involves only the explicit $x^{i}$ dependence in $F$ since any implicit dependence, via the $(\zeta, \bar{\zeta})$, does not enter into the definition of $p_{i}$ because of Eq. (69).) Now depending on which case, $\# 1,2$ or 3 is relevant, we eliminate two of the five variables $\left(x^{i}, \zeta, \bar{\zeta}\right)$, in the $p_{i}=\frac{\partial F}{\partial x^{i}}\left(x^{i}, \zeta, \bar{\zeta}\right)$. This leaves the result that the six coordinates of $T^{*} M$, can be expressed in terms of three parameters, thus defining a three dimensional submanifold in each of the cases;

- In case $1, p_{i}=P_{i}\left(x^{i}\right), \quad x^{i}=x^{i}$; the three parameters $\left\{x^{i}\right\}=\chi^{\alpha}$.

- In case $2, p_{i}=P_{i}\left(x^{A}, \bar{\zeta}\right), \quad x^{A}=x^{A}, \quad x^{J}=X^{J}\left(x^{A}, \bar{\zeta}\right)$; the three parameters $\left\{x^{A}, \bar{\zeta}\right\}=\chi^{\alpha}$.

- In case $3, p_{i}=P_{i}\left(x^{J}, \zeta, \bar{\zeta}\right), \quad x^{A}=X^{A}\left(x^{J}, \zeta, \bar{\zeta}\right), \quad x^{J}=x^{J}$; the three parameters $\left\{x^{J}, \zeta, \bar{\zeta}\right\}=\chi^{\alpha}$. 
To simplify the discussion, we have referred to the three parameters, in each of the cases, simply as $\chi^{\alpha}$. In each case we thus have

$$
x^{i}=X^{i}\left(\chi^{\alpha}\right), \quad p_{i}=P_{i}\left(\chi^{\alpha}\right) .
$$

Of immediate relevance to us is the projection (Lagrange map) of the Lagrange submanifold into the configuration space which becomes in each of the cases, $x^{i}=X^{i}\left(\chi^{\alpha}\right)$ or

- In case $1, x^{i}=x^{i}$, trivial diffeomorphism

- In case $2, x^{A}=x^{A}, \quad x^{J}=X^{J}\left(x^{A}, \bar{\zeta}\right)$

- In case $3, x^{A}=X^{A}\left(x^{J}, \zeta, \bar{\zeta}\right), \quad x^{J}=x^{J}$

The caustics of this problem are the regions in the configuration space where the mappings \#1, 2 or 3 have rank two or one; i.e., where the Jacobian of the mapping vanishes. They occur when the determinant $\hat{D}=0$. The inverse image to the caustics in the parameter space are referred to as the critical points of the Lagrange map. It is clear that in case 1, the Jacobian is one and rank reduction can only occur in cases 2 and 3.

This treatment of caustics can be extended into the full four-space by eliminating, in the expression for $t=T\left(f ; x^{i}, \zeta, \bar{\zeta}\right)$, or in the implicit version, $f=F\left(t, x^{i}, \zeta, \bar{\zeta}\right)$, two of the five variables $\left(x^{i}, \zeta, \bar{\zeta}\right)$ via the cases $\# 1,2,3$. This results in $t$ now being a function of the three parameters, $\chi^{\alpha}$. Though we will not need the full theory here, this construction leads to a seven dimensional manifold, $\left(t, x^{i}, p_{i}\right)$ \{an example of a contact manifold $\}$ and a threedimensional submanifold of the contact manifold (a Legendre submanifold) defined by

$$
t=T\left(\chi^{\alpha}\right), \quad x^{i}=X^{i}\left(\chi^{\alpha}\right), \quad p_{i}=P_{i}\left(\chi^{\alpha}\right)
$$

as well as the Legendre mapping from the Legendre submanifold, to spacetime, $\left(t, x^{i}\right)$

$$
\left\{t, x^{i}, p_{i}\right\}\left(\chi^{\alpha}\right) \Rightarrow t=T\left(\chi^{\alpha}\right), \quad x^{i}=X^{i}\left(\chi^{\alpha}\right)
$$

a three-surface in space-time - the "big wavefronts" in Arnold 's language - in our case a null surface. The singularities of the map Eq.(74), where the rank 
drops below three, are the null surface singularities. These singularities, at fixed $t$, are the ("small") wavefront singularities of the previous section.

We now return to the question of the determination of the function $f=$ $F\left(t, x^{i}, \zeta, \bar{\zeta}\right)$ of Eq. $(\overline{66})$ for use in the study of null surfaces. Our choice will be, from Eq.(11),

$$
F\left(t, x^{i}, \zeta, \bar{\zeta}\right)=S^{* *}\left(t, x^{i}, \zeta, \bar{\zeta}\right) \equiv x^{a} \ell_{a}(\zeta, \bar{\zeta})+\alpha(\zeta, \bar{\zeta})
$$

There are three independent reasons for this choice;

1. It was the method of generating an arbitrary null surface from the complete solution, $x^{a} \ell_{a}(\zeta, \bar{\zeta})$; see Sec. II..

2. It was the method for the construction of a null surface such that the generators were orthogonal to a given two-surface; see Sec. III..

3. It arises from a variant of Fermat's Principle of stationary time: Consider a timelike worldline, $\mathfrak{L}$, (in a Lorentzian space-time) of the form, in local coordinates, $\left(x^{i}=\right.$ constant, $t$ varies $)$ and a two-dimensional spacelike surface, $\mathfrak{s}(\zeta, \bar{\zeta})$. Assume, locally, that from every point of $\mathfrak{s}(\zeta, \bar{\zeta})$ there is a null geodesic that reaches $\mathfrak{L}$ at a time $t=T\left(x^{i}, \zeta, \bar{\zeta}\right)$, then $t$ is extremized by those curves that are normal to $\mathfrak{s}(\zeta, \bar{\zeta})$. This result (which will be described in detail elsewhere) follows from Schrödinger's derivation 19 of the gravitational frequency shift.

Note that the rank condition on the matrix, Eq.(70a) is satisfied by direct calculation.

From the discussion of generating families, we see that the treatment of the null surfaces that we proposed, in Secs. II and III, namely to solve for the $\zeta=\Upsilon(x, y, z)$, was really only valid for Case 1 , but we actually used a version of Case 3 where the additional parameter $r$ was introduced in order not to single out any particular cartesian coordinate. Case 1 broke down precisely on the caustic, given by

$$
r^{2}=\gamma^{2} \alpha \bar{\partial}^{2} \alpha
$$

which is where Cases 2 and 3 must be applied.

What follows is a straightforward application of Case 3 of these ideas on one patch. For completeness we repeat some of the earlier steps 
Starting with

$$
u=S^{* *}\left(x^{a}, \zeta, \bar{\zeta}\right)=x^{a} l_{a}(\zeta, \bar{\zeta})+\alpha(\zeta, \bar{\zeta})
$$

then

$$
\begin{aligned}
\partial S^{* *} & =\frac{1}{\sqrt{2}(1+\zeta \bar{\zeta})}\left[\bar{\zeta}^{2} W-\bar{W}-2 z \bar{\zeta}\right]+\succsim \alpha, \\
\bar{\partial} S^{* *} & =\frac{1}{\sqrt{2}(1+\zeta \bar{\zeta})}\left[\zeta^{2} \bar{W}-W-2 z \zeta\right]+\bar{\partial} \alpha,
\end{aligned}
$$

with $W=(x+i y)$. From $\partial S^{* *}=0$ and $\bar{\partial} S^{* *}=0$, we obtain, (from Case 3, where $x^{A}$ are $(x, y)$ or $\left.(W, \bar{W})\right)$ that

$$
\begin{aligned}
W & =\frac{\sqrt{2}\left(\bar{\partial} \alpha+\zeta^{2} ð \alpha\right)-2 z \zeta}{1-\zeta \bar{\zeta}}, \\
\bar{W} & =\frac{\sqrt{2}\left(ð \alpha+\bar{\zeta}^{2} \bar{\partial} \alpha\right)-2 z \bar{\zeta}}{1-\zeta \bar{\zeta}},
\end{aligned}
$$

From Eq.(77) we have that

$$
\begin{aligned}
& p_{x}=\frac{\partial S^{* *}}{\partial x}=-\frac{\zeta+\bar{\zeta}}{\sqrt{2}(1+\zeta \bar{\zeta})} \\
& p_{y}=\frac{\partial S^{* *}}{\partial y}=\frac{i(\zeta-\bar{\zeta})}{\sqrt{2}(1+\zeta \bar{\zeta})}, \\
& p_{z}=\frac{\partial S^{* *}}{\partial z}=\frac{(1-\zeta \bar{\zeta})}{\sqrt{2}(1+\zeta \bar{\zeta})}
\end{aligned}
$$

Taking $p=\sqrt{2}\left(p_{x}+i p_{y}\right)$, we obtain

$$
p=-\frac{2 \zeta}{1+\zeta \bar{\zeta}}
$$

The Lagrange submanifold, parametrized by $(z, \zeta, \bar{\zeta})$, is give by

$$
\begin{aligned}
z & =z \\
W & =\frac{\sqrt{2}\left(\bar{\partial} \alpha+\zeta^{2} \partial \alpha\right)-2 z \zeta}{1-\zeta \bar{\zeta}}
\end{aligned}
$$




$$
\begin{aligned}
\bar{W} & =\frac{\sqrt{2}\left(ð \alpha+\bar{\zeta}^{2} \bar{\partial} \alpha\right)-2 z \bar{\zeta}}{1-\zeta \bar{\zeta}} \\
p & =-\frac{2 \zeta}{1+\zeta \bar{\zeta}} \\
\bar{p} & =-\frac{2 \bar{\zeta}}{1+\zeta \bar{\zeta}} \\
p_{z} & =\frac{(1-\zeta \bar{\zeta})}{\sqrt{2}(1+\zeta \bar{\zeta})}
\end{aligned}
$$

The projection to the configuration space is given by

$$
\begin{aligned}
z & =z, \\
W & =\frac{\sqrt{2}\left(\bar{\partial} \alpha+\zeta^{2} \partial \alpha\right)-2 z \zeta}{1-\zeta \bar{\zeta}}, \\
\bar{W} & =\frac{\sqrt{2}\left(ð \alpha+\bar{\zeta}^{2} \bar{\partial} \alpha\right)-2 z \bar{\zeta}}{1-\zeta \bar{\zeta}} .
\end{aligned}
$$

The critical points of the Lagrange map are obtained from the condition that the Jacobian

$$
J=\frac{\partial(z, W, \bar{W})}{\partial(z, \zeta, \bar{\zeta})}
$$

vanishes. The vanishing of $J$ is equivalent to $D=r^{2}-\partial^{2} \alpha \bar{\partial}^{2} \alpha=0$.

To construct the Legendrian submanifold (in the seven dimensional contact space, $\left.\left(t, x^{i}, p_{i}\right)\right)$ we take the generating family $u=S^{* *}\left(x^{a}, \zeta, \bar{\zeta}\right)$ where $u$ is constant and solve for $t$ expressing the contact coordinate $t$ in terms of the three parameters $(z, \zeta, \bar{\zeta})$ by

$$
t=\frac{\zeta \bar{W}+\bar{\zeta} W-z(1-\zeta \bar{\zeta})}{(1+\zeta \bar{\zeta})}+\sqrt{2}[u-\alpha(\zeta, \bar{\zeta})]
$$

with

$$
\begin{aligned}
W & =\frac{\sqrt{2}\left(\bar{\partial} \alpha+\zeta^{2} \partial \alpha\right)-2 z \zeta}{1-\zeta \bar{\zeta}} \\
\bar{W} & =\frac{\sqrt{2}\left(ð \alpha+\bar{\zeta}^{2} \bar{\partial} \alpha\right)-2 z \bar{\zeta}}{1-\zeta \bar{\zeta}} .
\end{aligned}
$$


The full Legendre submanifold is then given by Eqs. (82), (83), (84), (85), (86), (87) and (90).

Note that this entire construction, using Case $\# 3$, was valid where $1-$ $\zeta \bar{\zeta} \neq 0$ (or equivalently where $p_{z} \neq 0$ ). To include the region where $p_{z}=0$, a different choice of parametrization would be necessary, e.g. $(x, \zeta, \bar{\zeta})$, which is valid in the region where $p_{x} \neq 0$ or $(y, \zeta, \bar{\zeta})$, valid where $p_{y} \neq 0$.

Using the example $\# 2$, from Sec. IV, given by

$$
S^{* *}\left(x^{a}, \zeta, \bar{\zeta}\right)=x^{a} l_{a}(\zeta, \bar{\zeta})+\alpha(\zeta, \bar{\zeta}), \alpha=-\frac{(1-\zeta \bar{\zeta})(\zeta+\bar{\zeta})}{(1+\zeta \bar{\zeta})^{2}}
$$

with

$\partial S^{* *}=0 \Leftrightarrow\left\{x\left(-1+\bar{\zeta}^{2}\right)+i y\left(1+\bar{\zeta}^{2}\right)-2 z \bar{\zeta}\right](1+\zeta \bar{\zeta})=\sqrt{2}\left[1+\bar{\zeta}^{3} \zeta-3 \bar{\zeta}(\zeta+\bar{\zeta})\right]$

and

$\bar{\partial} S^{* *}=0 \Leftrightarrow\left\{x\left(-1+\zeta^{2}\right)-i y\left(1+\zeta^{2}\right)-2 z \zeta\right](1+\zeta \bar{\zeta})=\sqrt{2}\left[1+\zeta^{3} \bar{\zeta}-3 \zeta(\zeta+\bar{\zeta})\right]$

one could try to solve for different pairs from the set $(x, y, z, \zeta, \bar{\zeta})$. When $D \neq 0$ one could always solve for $(\zeta, \bar{\zeta})$, though in general there would be more than one solution; i.e., for fixed $(x, y, z)$ there would in general be more than one ray going thru that space-point, either at the same or at different times. Alternately one could try to solve in different regions for $(x, y),(y, z),(z, x)$, etc. Solving for $(x, y)$ or $W$ we have that the Lagrange map (from the $(z, \zeta, \bar{\zeta})$ parameter space) becomes

$$
\begin{aligned}
W & =\frac{\sqrt{2}\left(2 \zeta^{2}+4 \zeta \bar{\zeta}-1-\zeta^{2} \bar{\zeta}^{2}\right)-2 z \zeta(1+\zeta \bar{\zeta})}{1-\zeta^{2} \bar{\zeta}^{2}} \\
\bar{W} & =\frac{\sqrt{2}\left(2 \bar{\zeta}^{2}+4 \zeta \bar{\zeta}-1-\zeta^{2} \bar{\zeta}^{2}\right)-2 z \bar{\zeta}(1+\zeta \bar{\zeta})}{1-\zeta^{2} \bar{\zeta}^{2}}
\end{aligned}
$$

which in turn becomes the Legendre map when the contact coordinate $t$ is added in;

$$
t=\sqrt{2}\left\{u+\frac{1}{(1-\zeta \bar{\zeta})(1+\zeta \bar{\zeta})^{2}}\left[4 \zeta \bar{\zeta}(\zeta+\bar{\zeta})-\frac{z}{\sqrt{2}}\left(1+3 \zeta \bar{\zeta}+3 \zeta^{2} \bar{\zeta}^{2}+\zeta^{3} \bar{\zeta}^{3}\right)\right]\right\}
$$


Though none of the details of this analysis is particularly enlightening, it nevertheless shows how in principle one constructs the Lagrange submanifold and the Lagrange map even in the presence of the caustics.

\section{Families of Foliations}

In this section we will generalize, in the following sense, the ideas of Section II. Recently there has been a reformulation of General Relativity, referred to as the Null Surface Formulation (NSF) where the basic idea has been to use a family (a spheres worth) of null foliations of space-time, so that there are a spheres worth of null surfaces passing thru each point of spacetime.. These surfaces are described as the level surfaces of the function

$$
u=Z\left(x^{a}, \zeta, \bar{\zeta}\right)
$$

$x^{a}$ are the local space-time coordinates and $(\zeta, \bar{\zeta})$ are the complex stereographic coordinate on the sphere which labels the family of foliations. The function $\mathrm{Z}$, for every fixed value of $(\zeta, \bar{\zeta})$, satisfies the eikonal equation,

$$
g^{a b} \partial_{a} Z \partial_{b} Z=0
$$

Knowing these families of foliations one can construct the (conformal) metric in terms of $Z$. The idea was then to express the Einstein equations in terms of these surfaces, i.e., in terms of $Z$ and a conformal factor. Though this was successfully accomplished, a technical difficulty in fully understanding the equations arose due to the fact that the null surfaces developed singularities (caustics) and self-intersections. It was clear that the development of caustics was a generic feature of the equations but it was not at all clear how to see and study their existence directly in terms of the function $Z\left(x^{a}, \zeta, \bar{\zeta}\right)$ and its derivatives. In this section, we will study, in flat space, the construction of such families and show explicitly how to calculate the structure of the null surface singularities (the caustics and wave-front singularities) directly in terms of the $\mathrm{Z}$ function.

Locally (up to first derivatives) there is no direct curvature involvement in the eikonal equation, so that the form of the caustics in terms of $Z$ should apply equally in curved space as in Minkowski space. The results obtained here for Minkowski space will thus likely apply to the curved space situation.

Starting with the two-parameter family of plane waves used earlier, $Z_{0}\left(x^{a}, \zeta, \bar{\zeta}\right)=$ $x^{a} \ell_{a}(\zeta, \bar{\zeta})$, we will first construct a general two-parameter family of solutions 
to the flat-space eikonal equation, $Z\left(x^{a}, \mu, \bar{\mu}\right)$, with $(\mu, \bar{\mu})$ parametrizing the sphere; we then study the singularities and caustics of this new family.

We begin by generalizing Eq.(11), namely

$$
S^{* *}=x^{a} \ell_{a}(\zeta, \bar{\zeta})+\alpha(\zeta, \bar{\zeta})
$$

by writing $\alpha$ as a function on $S^{2} \mathrm{x} S^{2}$; i.e., as $\alpha=\alpha(\zeta, \bar{\zeta}, \mu, \bar{\mu})$ and then repeating the earlier procedure of setting to zero, the $\partial$ and $\bar{\partial}$ derivatives with respect to the $(\zeta, \bar{\zeta})$; i.e., considering

$$
u=Z^{* *}(x, \zeta, \bar{\zeta}, \mu, \bar{\mu})=x^{a} \ell_{a}(\zeta, \bar{\zeta})+\alpha(\zeta, \bar{\zeta}, \mu, \bar{\mu})
$$

and

$$
\partial_{\zeta} Z^{* *}=\bar{\partial}_{\zeta} Z^{* *}=0
$$

and then solving them (when possible) for $(\zeta, \bar{\zeta})$ obtaining

$$
\zeta=\Upsilon(x, y, z, \mu, \bar{\mu}), \quad \bar{\zeta}=\bar{\Upsilon}(x, y, z, \mu, \bar{\mu})
$$

so that when substituted into Eq.(99) we obtain the new family which depends on the choice of $\alpha(\zeta, \bar{\zeta}, \mu, \bar{\mu})$;

$$
Z\left(x^{a}, \mu, \bar{\mu}\right)=x^{a} l_{a}(\Upsilon, \bar{\Upsilon})+\alpha(\Upsilon, \bar{\Upsilon}, \mu, \bar{\mu}) .
$$

(Alternatively we could use the different cases of Sec. V, when one can not solve for $(\zeta, \bar{\zeta})$.) It is obvious from the previous discussion that Eq.(102) satisfies the eikonal equation for each fixed value of $(\mu, \bar{\mu})$. All we have done so far is create a new spheres worth of null foliations (wavefront families) of Minkowski space - different from the plane wave case of $S=x^{a} \ell_{a}(\zeta, \bar{\zeta})$. As in the earlier sections we could have analyzed the null surfaces for each value of $(\mu, \bar{\mu})$ separately but now in this generalization the null surfaces are smoothly connected to each other through the variable $(\mu, \bar{\mu})$ and it becomes of interest to see the development of the caustics via the variation of the $(\mu, \bar{\mu})$, or through the $(\mu, \bar{\mu})$ derivatives.

Remark 2 We will use, respectively, the notation $\left(\Varangle_{\mu}, \bar{\partial}_{\mu}\right)$ for the eth and ethbar derivatives with respect to the variables $(\mu, \bar{\mu})$ and $\left(\partial_{\zeta}, \bar{\partial}_{\zeta}\right)$ for the variables $(\zeta, \bar{\zeta})$. 
We begin by defining several derivatives of $Z$;

$$
\begin{gathered}
\omega=\partial_{\mu} Z, \bar{\omega}=\bar{\partial}_{\mu} Z \\
R=\bar{\partial}_{\mu} \partial_{\mu} Z \\
\Lambda=\partial_{\mu}^{2} Z, \bar{\Lambda}=\bar{\partial}_{\mu}^{2} Z .
\end{gathered}
$$

A level surface of Z, with fixed $(\mu, \bar{\mu})$, is ruled by null geodesics, whose tangent vectors are given by $\ell_{a}(\Upsilon, \bar{\Upsilon})$; A pencil of rays defined from a pair of geodesic deviation vectors (from a given geodesic) has an area $A$ that can be given 20 up to a proportionality by

$$
A=K \frac{\Omega^{-2}}{\sqrt{\left(1-\Lambda_{, 1} \bar{\Lambda}_{, 1}\right)}}
$$

where $K$ is a constant determined by the initial area and

$$
\Omega^{2}=\ell^{a} R,{ }_{a}
$$

and

$$
\Lambda_{, 1}=\Omega^{-2} \ell^{a} \Lambda_{,} .
$$

The derivation of Eq.(106) is lengthy and will not be given here. It will however be shown, in this case to be proportional to the area.

We now want to see the behavior of $\omega, \Lambda$ and $R$, as well as the area $A$, in the neighborhood of a caustic.

By direct calculation we have, from Eqs.(102 and 100), that

$$
\omega=\partial_{\mu} \alpha, \bar{\omega}=\bar{\jmath}_{\mu} \alpha,
$$

and hence is singularity free.

After a rather lengthy calculation, using Eqs.(99) and (100), we obtain

$$
\begin{aligned}
R= & \partial_{\mu} \bar{\partial}_{\mu} \alpha+\frac{1}{D}\left\{\left(\partial_{\zeta}{\partial_{\mu}}_{\mu} \alpha\right)\left[\left(\partial_{\zeta} \bar{\partial}_{\mu} \alpha\right)\left(\bar{\partial}_{\zeta}^{2} u\right)-\left(\bar{\partial}_{\zeta} \bar{\partial}_{\mu} \alpha\right)\left(\partial_{\zeta} \bar{\partial}_{\zeta} u\right)\right]\right. \\
& \left.+\left(\bar{\partial}_{\zeta} \partial_{\mu} \alpha\right)\left[\left(\bar{\partial}_{\zeta} \bar{\partial}_{\mu} \alpha\right)\left(\partial_{\zeta}^{2} u\right)-\left(\partial_{\zeta} \bar{\partial}_{\mu} \alpha\right)\left(\partial_{\zeta} \bar{\partial}_{\zeta} u\right)\right]\right\} .
\end{aligned}
$$

where 


$$
D=\left(\partial_{\zeta} \bar{\partial}_{\zeta} u\right)^{2}-\partial_{\zeta}^{2} u \bar{\partial}_{\zeta}^{2} u
$$

In a similar way we obtain

$$
\begin{aligned}
\Lambda= & \partial_{\mu}^{2} \alpha+\frac{1}{D}\left\{\left(\partial_{\zeta} \partial_{\mu} \alpha\right)\left[\left(\partial_{\zeta} \partial_{\mu} \alpha\right)\left(\bar{\partial}_{\zeta}^{2} u\right)-\left(\bar{\partial}_{\zeta} \partial_{\mu} \alpha\right)\left(\partial_{\zeta} \bar{\partial}_{\zeta} u\right)\right]\right. \\
& \left.+\left(\bar{\partial}_{\zeta} \partial_{\mu} \alpha\right)\left[\left(\bar{\partial}_{\zeta} \partial_{\mu} \alpha\right)\left(ð_{\zeta}^{2} u\right)-\left(\partial_{\zeta} \partial_{\mu} \alpha\right)\left(\partial_{\zeta} \bar{\partial}_{\zeta} u\right)\right]\right\} .
\end{aligned}
$$

First we see that for fixed values of $(\mu, \bar{\mu}), D$ from Eq. (109), is the same as in Eq. (32), namely $D=r^{2}-\partial_{\zeta}^{2} \alpha \bar{\partial}_{\zeta}^{2} \alpha$ and hence vanishes at the caustic. We can now see that $\omega$ is regular at the caustic while both $R$ and $\Lambda$ have singularities of the form $D^{-1}$ at the caustic.

In order to find the area $A$ we first need $R,,_{a}$ and $\Lambda_{,_{a}}$. After a lengthy calculation we obtain

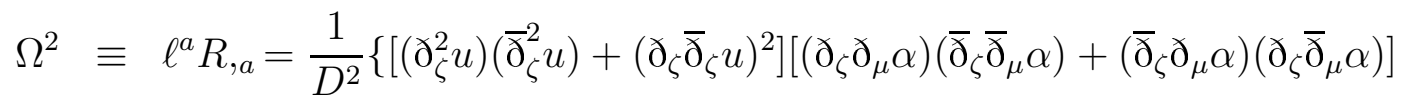

$$
\begin{aligned}
& \left.-2\left[\left(\partial_{\zeta} \partial_{\mu} \alpha\right)\left(\partial_{\zeta} \bar{\partial}_{\mu} \alpha\right)\left(\bar{\partial}_{\zeta}^{2} u\right)+\left(\bar{\partial}_{\zeta} \bar{\partial}_{\mu} \alpha\right)\left(\bar{\partial}_{\zeta} \partial_{\mu} \alpha\right)\left(\partial_{\zeta}^{2} u\right)\right]\left({\partial_{\zeta}}_{\zeta} u\right)\right\}
\end{aligned}
$$

and

$$
\begin{aligned}
\Omega^{2} \Lambda_{, 1} \equiv & \ell^{a} \Lambda_{a}=\frac{2}{D^{2}}\left\{\left[\left(\partial_{\zeta}^{2} u\right)\left(\bar{\partial}_{\zeta}^{2} u\right)+\left(\partial_{\zeta} \bar{\partial}_{\zeta} u\right)^{2}\right]\left(\partial_{\zeta} \partial_{\mu} \alpha\right)\left(\bar{\partial}_{\zeta} \partial_{\mu} \alpha\right)\right. \\
& \left.-\left[\left(\searrow_{\zeta} \partial_{\mu} \alpha\right)^{2}\left(\bar{\partial}_{\zeta}^{2} u\right)+\left(\bar{\partial}_{\zeta} \partial_{\mu} \alpha\right)^{2}\left(\partial_{\zeta}^{2} u\right)\right]\left(\partial_{\zeta} \bar{\partial}_{\zeta} u\right)\right\}
\end{aligned}
$$

Though it is not immediately obvious, from Eqs.(111) and (112) one can show that $\left(1-\Lambda_{, 1} \bar{\Lambda}_{, 1}\right)$ is proportional to $D^{2}$ or that $\left|\Lambda_{, 1}\right| \Rightarrow 1+O(D)$ at the caustic. From these results we have that

$$
A=K \frac{\Omega^{-2}}{\sqrt{\left(1-\Lambda_{, 1} \bar{\Lambda}_{, 1}\right)}}=\frac{ \pm K D}{\hat{K}}
$$

with

$$
\hat{K}=\left|ð_{\zeta} ð_{\mu} \alpha\right|^{2}-\left|\bar{\partial}_{\zeta} \partial_{\mu} \alpha\right|^{2} .
$$

From Eqs. (100) and (101), we have that $\hat{K}=\hat{K}(\Upsilon, \bar{\Upsilon})$ from which it can be shown that

$$
\ell^{a} \hat{K}_{a}=0
$$


i.e., $\hat{K}$ is constant along the geodesic flow. If we chose $K= \pm \hat{K}$ we have that $A=D$, in agreement with the area obtained in section III from geodesic deviation.

Several important observations can now be made:

1. In our particular case of flat space, we have seen that the quantities $R$ and $\Lambda$ diverge as $D^{-1}$ at the caustic. It appears virtually certain that this is a general result and remains true in a general curved space.

2. As was to be expected the Area of a pencil of null geodesics vanishes at the caustic. This is clearly true in general and the result here is a confirmation that the Eq.(106) really is the area formula.

3. The quantity $\Omega$ (which plays a central role in the NSF version of GR) diverges as $D^{-1}$ at the caustic.

4. Though $\Lambda$ diverges at the caustic, the absolute value of its weighted derivative

$$
\left|\Lambda,{ }_{1}\right|=\left|\Omega^{-2} \Lambda,{ }_{a} \ell^{a}\right|
$$

approaches one as $1-O(D)$. From this one sees that $\Lambda_{,} \ell^{a}$ diverges as $D^{-2}$.

\section{Discussion}

Our main interest in the study of wavefronts and their associated null surfaces, lies in our desire to understand and describe their singularity structure in curved Lorentzian manifolds and in particular to find the most appropriate variables and representations for their analysis. Though locally the classification of generic singularities and caustics is complete and is the same in both flat and curved spaces, 5 however, in general spaces, curvature effects are large and must eventually be taken into account for global questions. (For example, the structure of the lightcone in a curved space is very different from that of a lightcone in flat space.) The present work is intended to begin this study with the description of singular, global, asymptotically spherical, null surfaces in flat spaces. A follow-up second paper, will be devoted to the same issues as here but in asymptotically flat space-times. We will see that beginning with a two-parameter family of solutions of the eikonal equation analogous to the plane wave solutions of flat space-time - it will be possible to construct any other null surface and then analyze its singularity structure. In particular, it is possible to construct, in terms of the two parameter family, the light cone of any space-time point. These insights are important for 
applications of the null surface formulation of GR. 8 .

\section{Acknowledgment}

We would like to thank Carlos Kozameh for many very useful arguments and even for some peaceful conversations. Also we want to point out that there has been a fair amount of unwitting and uncoordinated overlap between the work of the present authors (in Secs. II and III) and the group of Bishop, Gomez, Lehner, Szilagyi and Winicour 18 Since the motivation, notation and final results of the work of the two groups are quite different, it has been decided to keep the work separate and publish independently.

We also thank the referee, Juergen Ehlers, for valuable improvements and corrections.

SF and EN thank the NSF for support under grant \#PHY 92-05109. GSO acknowledges the financial support from the Sistema Nacional de Investigadores and from the Consejo Nacional de Ciencia y Tecnologia (CONACyT) Mexico. 


\section{References}

${ }^{1}$ V. I. Arnold, S.M. Gusein-Zade, A. N.. Varchenko, Singularities of Differentiable Maps, Vol. I, Birkhauser, Boston, Basel, Stuttgart, (1985).

${ }^{2}$ V. I. Arnold, Catastrophe Theory, Springer-Verlag, Berlin, Heidelberg, NY, Tokyo, (1986).

${ }^{3}$ V. I. Arnold, Mathematical Methods of Classical Mechanics, SpringerVerlag, Berlin, Heidelberg, NY, (1980).

${ }^{4}$ V. I. Arnold, S.P. Novikov, eds., Dynamical Systems; Vol. IV, , SpringerVerlag, Berlin, Heidelberg, NY, (1990).

${ }^{5}$ Friedrich, H. and Stewart, J., Proc. Roy. Soc. A385, 345-371, (1983).

${ }^{6}$ W.Hasse, M. Kriele, and V. Perlick, Class. Quant.Grav. 13,1161 (1996)

${ }^{7}$ P. Schneider, J. Ehlers, E.E. Falco, Gravitational Lenses, Springer-Verlag, New YOrk, Berlin, Heidelberg, (1992).

${ }^{8}$ S. Frittelli, C. Kozameh, E. Newman, J. Math. Phys. 36, 4984, (1995).

${ }^{9}$ S. Frittelli, C. Kozameh, E. Newman, J. Math. Phys. 36 , 6397, (1995).

${ }^{10}$ R. Penrose and W. Rindler, Spinors and Space-Time, Cambridge University Press, Cambridge, England, (1994).

${ }^{11}$ E.T. Newman and K.P. Tod, Asymptotically Flat Space-Times, in General Relativity and Gravitation, Vol.2, Edited by A. Held, Plenum Publishing Corporation, (1980) and references cited therein.

${ }^{12}$ L. Landau and Lifschitz, Classical Mechanics, Pergamon Press, Headington Hill Hall, Oxford, 4 \& 5 Fitzroy Sq. London, W.1, (1960).

${ }^{13}$ R. Courant and D. Hilbert, Methods of Mathematical Physics, Interscience Publishers, New York-London, Vol.II, (1962) .

${ }^{14}$ C. Kozameh, E. Newman, J. Math. Phys., 24 ,2481,(1983).

${ }^{15}$ S.L. Kent and E. T. Newman, J.Math. Phys., 24,949 (1983).

${ }^{16}$ S. Frittelli, C. Kozameh, E. Newman, Phys Rev. D. 56 , 4729, (1997). 
${ }^{17}$ R.W.Lind and E.T. Newman, J.Math.Phys.,15,1103,(1974).

${ }^{18}$ A recent preprint by Bishop,Gomez, Lehner, Szilagyi and Winicour uses this same form of the metric for the study of numerical solutions of the Einstein equations.

${ }^{19}$ I. Schrodinger, Expanding Universes, Cambridge University Press, Cambridge, England, (1956).

${ }^{20}$ C. Kozameh, E. Newman, A Non-Local Approach to the Vacuum Maxwell, Yang-Mills and Einstein equations, in Topological Properties and Global Structure of Space-Time, eds P. Bergmann and V. de Sabbata, Plenum Press (1986) 